\title{
Propuesta de la estructura organizacional para un observatorio de investigación contable en Colombia
}

\author{
Proposal of the organizational \\ structure for an accounting research \\ observatory in Colombia
}

DOI: https://doi.org/10.17981/econcuc.41.2.2020.Org.5

Artículo de investigación.

Fecha de recepción: 07/02/2020

Fecha de devolución: 23/04/2020

Fecha de aceptación: 30/04/2020

Fecha de publicación: 08/05/2020

\section{Luis Alfonso González Feliciano}

Universidad Militar Nueva Granada

Bogotá, D.C. (Colombia)

luis.gonzalez@unimilitar.edu.co

\section{Resumen}

En anteriores escritos sobre el tema de los observatorios contables a nivel nacional, se ha evidenciado la necesidad de hacer una propuesta estructural que contenga elementos tanto en la parte de gestión como también en la parte organizacional para su montaje y puesta en marcha, además es de resaltar la importancia que ocupa la información en su manejo y asequibilidad para los investigadores, siendo la base sobre la que se apoya la investigación en cualquier área del conocimiento, y puesto que en este momento no existe un observatorio de investigación contable en Colombia que pueda ser tomado como ejemplo para implementación, se presenta en este escrito la propuesta para la parte organizacional, que es un avance en la idea de montar un ente con características propias de su definición, se han tomado múltiples ejemplos de observatorios de diferentes disciplinas en distintos países y también el argumento teórico de diferentes autores desde la perspectiva administrativa, los cuales aportan conocimiento para cada uno de los elementos presentados siendo importantes para integrar y tener en cuenta a la hora de su estructuración y funcionamiento. Se concluye que la propuesta puede ser usada por todo tipo de organización universidades, empresas, grupos de investigación, etc.

Palabras clave: Guía; observatorio contable; estructura organizacional

\begin{abstract}
In previous writings on the subject of accounting observatories at the national level, it has been evidenced the need to make a structural proposal that contains elements both in the management part as well as in the organizational part for its assembly and start-up, in addition to highlight the importance of information in its management and affordability for researchers, being the basis on which research is supported in any area of knowledge, and since at this time there is no accounting research observatory in Colombia that can be taken as an example for implementation, the proposal for the organizational part is presented in this writing, which is an advance in the idea of setting up an entity with characteristics of its definition, multiple examples of observatories of different disciplines in different countries have been taken and also the theoretical argument of different authors from the administrative perspective, which contributed a knowledge for each of the related elements being important to integrate and take into account when structuring and operating. It is concluded that the proposal can be used by all types of organizations, universities, companies, research groups, etc.

Keywords: Guide; accounting observatory; Organizational
\end{abstract} structure 


\section{INTRODUCCIÓN}

Proponer la estructura organizacional de un observatorio contable es la parte fundamental en este escrito, es una propuesta practica que seguramente podría relacionarse con la minería de datos o el Big data que está revolucionando el mundo del conocimiento, se podría tomar en otro escrito la propuesta para el apoyo a la parte estratégica y así queda consolidada toda la guía para el montaje del proyecto y evita incrementar la extensión de este.

El escrito se soporta en evidencias de anteriores investigaciones realizadas por el autor que demuestran la inexistencia de una organización con estas características de observatorio en el área contable en Colombia. La necesidad de que exista un observatorio está fundamentada en la experiencia de búsqueda de información para investigar en el área contable, la dispersión en la búsqueda de información sobre tendencias de investigación a nivel nacional; antecedentes, información actualizada sobre líneas, tendencias en investigación, avances en el área contable en el país (Bejarano, González y Matallana, 2017), es verdad que si existen trabajos de muchos investigadores o grupos que de manera particular cubren algunas temáticas y tiempos acorde a sus intereses, sin embargo, facilitaría el avance de los investigadores el poseer una información mejor organizada. (Paredes-Chacín, 2017; Paz, Harris y Franco, 2016).

Para esta investigación se han tomado variadas fuentes bibliográficas que soportan desde la teoría las partes de la guía que se presentan en la misma y de cada uno de sus elementos, además se aplica la revisión de múltiples observatorios ya en funcionamiento en diferentes disciplinas tanto a nivel nacional como internacional para evaluar especialmente sus estructuras para aprender de los elementos necesarios en el funcionamiento.

Se resalta que a nivel nacional los diferentes intentos de universidades por crear un observatorio han sido infructuosas, se encontraron falencias tanto en sus estructuras como en la forma que definían observatorio, las cuales quedaron expuestas por medio de visitas y entrevistas, en ese momento se explicó de manera sencilla pero completa: la definición de observatorio y su importancia de contener todos los elementos que le permiten que se pueda nombrar así, allí también se sustenta desde lo teórico cada una de estas definiciones y elementos.

Se aborda el tema de la siguiente forma: primero se resumen algunos antecedentes con relación a las estructuras organizacionales de diferentes observatorios en contextos internacionales y nacionales, luego se presenta el proceso realizado en la metodología utilizada, en la tercera parte están los componentes estructurales, en seguida se encuentran las actividades generales propuestas, para finalmente presentar la estructura organizacional general por áreas de forma gráfica, las sugerencias, consideraciones y conclusiones.

De la estructura organizacional quedara cada uno de los detalles de las áreas misionales, y al finalizar este escrito el lector entienda los elementos necesarios y básicos en la formulación de este proyecto. 


\section{Algunos antecedentes}

Existen guías nacionales como la guía para la conformación de observatorios de derechos humanos, proyecto que estuvo en cabeza del presidente Álvaro Uribe Vélez, ella contiene en su estructura: sistemas de información, definición, cuáles son las redes o fuentes de información con que trabaja, cómo se debe manejar y clasificar la información, variables para la creación de formularios o aplicativos que sirven para consolidar información, manejando ítems como; nombre, sexo, clasificación, etc., formas de almacenar la información, procesamiento de información, salida de información, con otros elementos necesarios.

Los ejemplos internacionales revisados en el artículo titulado Componentes y variables de los observatorios contables existentes a nivel superior en Colombia, Bejarano, González y Matallana (2017), además de guías y bibliografía relacionadas con distintas disciplinas, fueron confrontados en sus características estructurales y estas variables se fueron consolidando, generando comunes denominadores durante las revisiones, muchos de ellos demostraron poseer características básicas de acuerdo a la Figura 1, las cuales permiten ir depurando el esquema pretendido, algo definitivo es el haber encontrado que todas las fuentes, poseían unos aspectos que diferencian en definitiva a los observatorios de los grupos de investigación, semilleros y demás organismos de carácter investigativo más especializado.

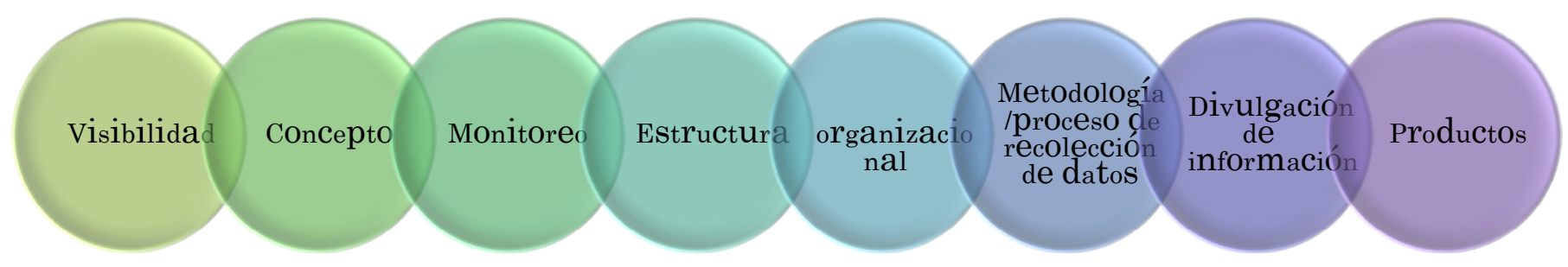

Figura 1. Características básicas de los observatorios. Fuente: Elaboración propia con variables de referencia para la identificación de observatorios, tomadas de diferentes fuentes y complementadas con elementos administrativos con base en Moreno y Del Pilar (2009).

La visión académica de observar para investigar o viceversa, es una de las actividades fundamentales en el observatorio, sin embargo, para que este proceso se lleve a cabo de una manera más ordenada haciendo parte de una información consolidada y de fácil acceso, es necesario la definición de los componentes administrativos de estructura, que facilitan el manejo de recursos, información, recursos financieros y otros que se irán presentando más adelante.

Las entidades de tipo observatorio en la disciplina contable a nivel nacional, no existen, son todos grupos de investigación y cabe anotar que algunas entidades de tipo contable como Colegio de Contadores Públicos de Colombia, Conpucol o el Consejo Técnico de la Contaduría pública no evidencian tener proyectos cercanos o que se encuentren en fase de desarrollo o en implementación sobre guías para implementar un observatorio. 
A pesar de las buenas labores por parte de las instituciones de educación superior en la promoción de conocimiento contable e investigación, y aun con la participación de las universidades en los convenios interuniversitarios o en los institutos gremiales, como es el caso del Instituto Nacional de Contadores o el Colegio Colombiano de contadores, no se ha logrado crear un espacio que promueva de manera eficiente y efectiva el conocimiento del estado de los productos de investigación contable en el país, además no existe un canal fuerte de difusión de información que concentre, genere y pueda ser un motor de desarrollo económico el cual sería facilitado desde los resultados en las investigaciones del área contable (Organización para la Cooperación y el Desarrollo Económico-OCDE, 2016).

\section{Metodología}

La metodología es de tipo cualitativo y descriptivo, observando las características donde se focalizan las estructuras de observatorios nacionales como también algunos internacionales, se compone de aproximadamente (30) treinta revisiones de observatorios de diversas áreas del conocimiento, lo anterior se complementara con elementos de la metodología del marco lógico de Ortegón, Pacheco y Prieto (2005) en el cual se involucra en el análisis por medio de; encuestas, visitas y entrevistas, donde se tomaron (154) ciento cincuenta y cuatro universidades nacionales que poseían observatorios, todo esto con el fin de describir una estructura clara desde lo organizacional para este proyecto, adicionalmente en este artículo se evaluaron además seis guías completas de observatorios de diferentes disciplinas, y más de (12) doce autores citados a lo largo del desarrollo de este escrito que hablan sobre elementos relacionados con la estructura de un observatorio, algunos de ellos contienen temas como por ejemplo: observatorios de investigación, productos de investigación, gestión de la información, redes de cooperación, sistemas de información, estructuras organizacionales, entre otros, en resumen las fuentes consultadas con el fin de evaluación se pueden verificar en la Figura 2:

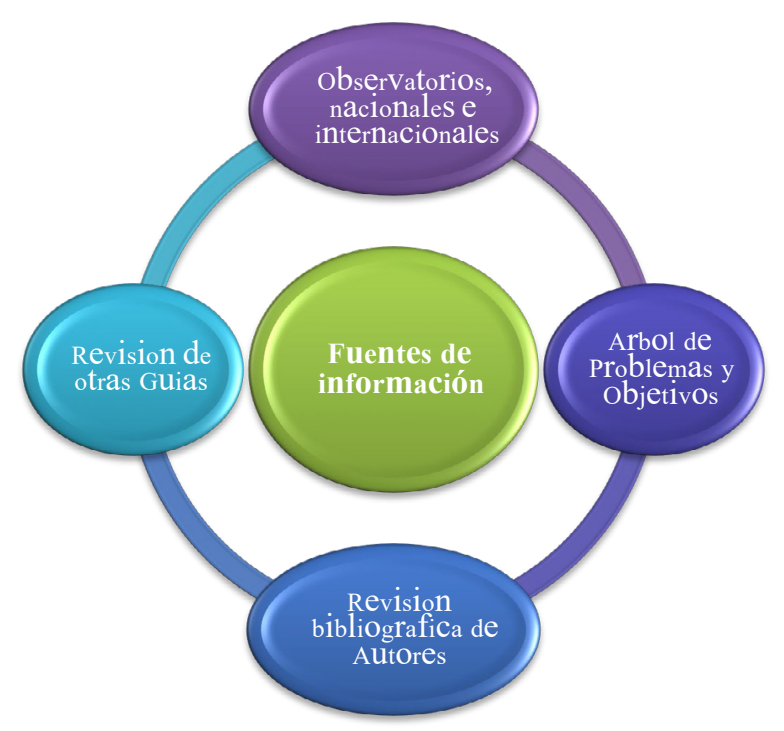

Figura 2. Fuentes consultadas para evaluar las partes de la guía. Fuente: Elaboración propia a partir de la metodología. 
El mantenimiento en movimiento de las redes de cooperación ha sido una de las principales debilidades encontradas, ya que la información generada desde los convenios no es sostenible en el tiempo, sin el flujo constante de la información no existe materia prima para el estudio de las observaciones de cualquier tipo en el ámbito contable, esta falla se ha evidenciado tanto en el árbol de problemas, como también en las visitas realizadas, resultados que quedaron consignados en los primeros artículos del autor, más adelante se darán algunas recomendaciones desde la estructura para su fortalecimiento.

\section{Guías ya estructuradas de Observatorios en diferentes temáticas}

A continuación, se presentan seis (6) guías ya estructuradas de observatorios que posiblemente se encuentren en funcionamiento, en ellas se muestran componentes explícitos, que de manera ordenada hacen parte de observatorios en múltiples contextos temáticos. (Tabla 1)

TABLA 1.

Consolidado de Guías sobre conformación de observatorios de otras temáticas

Guía para la conformación de Observatorios de Derechos H
umanos y DIH de la vicepresidencia Gobierno de la República,
Agosto de 2006, Colombia (Presidencia de la República de
Colombia, 2006).
Bases para la contrucción de observatorios territoriales de
juventud, Programa presidencial para el sistema nacional de
juventud (Colombia Joven), Noviembre 2012 (Presidencia de la
República Colombia, 2012).
Análisis y propuesta de implementación de un observatorio
TIC para un conjunto de mipymes de la localiadd de
Usaquén(Bogota en la universidad de San Buenaventura)
(Torres y Martínez, 2014).
Manual para la presentación de proyectos Fondoempleo, 2010,
Colombia (Müller, 2010).
Observatorio SIC: análisis de una iniciativa de comunicación
basada en el expertise del servicio de información y
conocimiento de ESADE, noviembre 2007, España (Soler y
Catalá, 2007).
“Observatorio de turismo". Un espacio de análisis y
Comunicación como contribución para el desarrollo del
Turismo en el Ecuador, 2013 (Carrera y López, 2013).

Fuente. Elaboración propia a partir de las guías consultadas.

De las anteriores guías se tomarán algunos elementos de cada una de ellas, conformando y complementando estructuralmente la mejor propuesta, la cual se ira conformando los siguientes apartados. 


\section{FundAMENTOS TEÓRICOS}

\section{Elementos estructurales para el observatorio contable en Colombia}

La búsqueda desde diferentes referentes teóricos, parte con Moyares e Infante (2016) quienes relacionan su mirada de los observatorios desde la tecnología, en la actualidad la tecnología como herramienta para el manejo de la información se hace primordial, los modelos de información y la tecnología debe ir de la mano en contextos relacionados con la educación superior (Acurero, Ziritt y Chumaceiro, 2017; Hernández y Buitrago, 2017; Hernández, Meléndez, Chumaceiro y Aguilar, 2017).

El papel de la información en los observatorios es clave ya que permite mantener la unidad y el flujo de insumos, un observatorio sin información de calidad por si solo quedaría excluido en la sociedad del conocimiento, así entonces el factor de éxito en un observatorio es la creación, mantenimiento y fortalecimiento de las redes de cooperación entre instituciones. Para Ravina-Ripoll, Ahumada-Tello y Hernández de Velasco (2018), al igual que Méndez, Jiménez y Cervantes (2016), las redes de investigación y cooperación son muy importantes para potencializar factores como la calidad de las publicaciones, si se logra mantener el flujo de información y se estructura con calidad, con un alto nivel de difusión, de observación de los resultados de investigaciones, se esperaría que el estado de avance en las investigaciones de temáticas contables se viera fortalecido, a su vez la disciplina contable mejorara los sistemas educativos, el impulso en investigación por ende se tendrá que ver reflejado en el desempeño profesional.

Para desarrollar las actuales condiciones del país en información investigativa contable, se debe valorar la relación entre sistemas de información y su labor dentro de los observatorios, por esto se revisan los tipos de SI que se podrían aplicar dentro del observatorio, se toman tres (3) diversas relaciones, las que se enfocan a las transacciones con el fin de recolectar y manejar grandes volúmenes de información de forma adecuada, las de soporte a tomas de decisiones que se enfocan más a interactuar con el usuario y a pensar en satisfacerlo y las estratégicas que visionan a futuro la empresa, buscando ventajas competitivas, los tres enfoques son aplicables al caso (González, 2007).

Por otra parte, Machado y Zapata (2016), indican que la utilidad de la información contable en el contexto en el cual se encuentre, genera un efecto catalizador a la sociedad para su avance, la calidad es primordial y luego de ser entregada a los grupos de interés, ellos pueden utilizarla de manera inesperada en diferentes formas creativas que aportan al desarrollo, es decir infinidad de posibilidades de mejora al ser aplicada, así se valoran y aplican los sistemas de información dentro del proceso.

Existen variadas propuestas e ideas plasmadas en diferentes documentos sobre la puesta en marcha de un observatorio, sin embargo, es curioso que aun cuando existen los conocimientos, no han podido ser llevados a la práctica en nuestro país y en nuestra disciplina, existe un documento divulgado por la Universidad Santo Tomás (Observatorio Colombiano de educación contable, 2012). Con buenas ideas que al ser relacionadas con otros autores como Pinzón (2012), resulta un aporte de ideas valioso 
que explica por qué el mejoramiento de la calidad de la información contable disponible, está relacionado con el fin de formar mejores contadores Públicos, se puede afirmar que producir información de calidad en el área académica, y al ser utilizada por los investigadores y académicos, sustentará conocimiento nuevo e innovadores proyectos, basados en los sistemas de información dentro de la estructura.

La visibilidad y la capacidad de difundir el conocimiento, si ese sistema de información tecnificado no produce un impacto y no llega a la sociedad objetivo, no existirá una conexión y propagación de conocimiento que sea útil y aprovechada por los diferentes interesados de la disciplina contable.

Los observatorios son una opción clara que permite que los actores de un sector incremente su desarrollo en el área de enfoque, sea económica, política, científica, etc. Vallejo, Echavarría y Uribe (2009) nos muestran que existen infinidad de estructuras ya en funcionamiento, el problema se encuentra en poder adaptarlas y ponerlas en funcionamiento en nuestro saber contable.

El personal que integra la entidad elemento primordial y al respecto Bianco, Lugones, Peirano y Salazar (2016) nos dan guía que en esta era, el proceso productivo requiere de personal adecuado, el cual se encuentre en la capacidad de producir bienes diferenciados y no solo producción, en este caso información, es por esto que el componente humano es la principal fuente de apoyo en cualquier proceso y más aún en el ámbito académico, el criterio, los procesos investigativos no se podrían hacer con la sola inversión tecnológica, administrativa o financiera, es importante el entendimiento del aporte y contribución de docentes o investigadores, teniendo en cuenta el perfil del personal y de cada uno de sus integrantes basado en las tareas que van a desempeñar.

La elaboración de una estructura administrativa también se puede evaluar apoyados en la visión de un autor como Shein (citado por Barrios, 2009), quien ve la organización con visión sistémica, desde un sistema abierto que es complejo y posee muchas partes y medios, todos con unas metas por alcanzar en cada uno de sus niveles y complejidades, siempre desarrollándose en un medio que evoluciona y que a su vez genera nuevas acomodaciones dentro de la estructura, el trabajo es adaptarse al medio y fortalecerse en sus procesos internos.

En el país, la Presidencia de la República (2006), ya posee muchos proyectos de observatorios en funcionamiento y también guías como la de derechos humanos que es herramienta útil para la construcción, análisis, diagnóstico y generación de documentos enfocados hacia observatorios en lo contable, además ejemplo claro de la importancia de este tipo de estructuras y el aporte que ellas pueden lograr en nuestro país.

Se encuentran también otros factores que se deben adaptar y hacer parte de los observatorios, en documentos de Moreno y Del Pilar (2009) y De la Vega (2007), quienes evalúan y revisan diversos tipos de observatorios que son funcionales en múltiples disciplinas, conocimientos que enseñan cómo se conforman y se dividen sus áreas, allí se puede evidenciar la planeación en gestión de procesos y los resultados de participación y engranaje en cada área, esto aporta al proceso. 
El tipo de organización en cuanto a su estructura formal o legal debe ser tenida en cuenta, ya que al ser sin ánimo de lucro, genera múltiples particularidades al respecto, es positivo el ser sin ánimo de lucro puesto que, como indica Donati (1997), se percibe de manera importante el objetivo social, en el cual, quien participa lo hace con el ánimo de contribuir a la sociedad, el conocimiento y los aportes investigativos se desvían del pensamiento meramente de negocio de producir ganancias monetarias, posee tanto de positivo como también en contra porque se hace más difícil el conseguir el interés de participación de los sectores, en un artículo de Robles (2015), en el cual determina algunos de estos problemas (Figura 3).

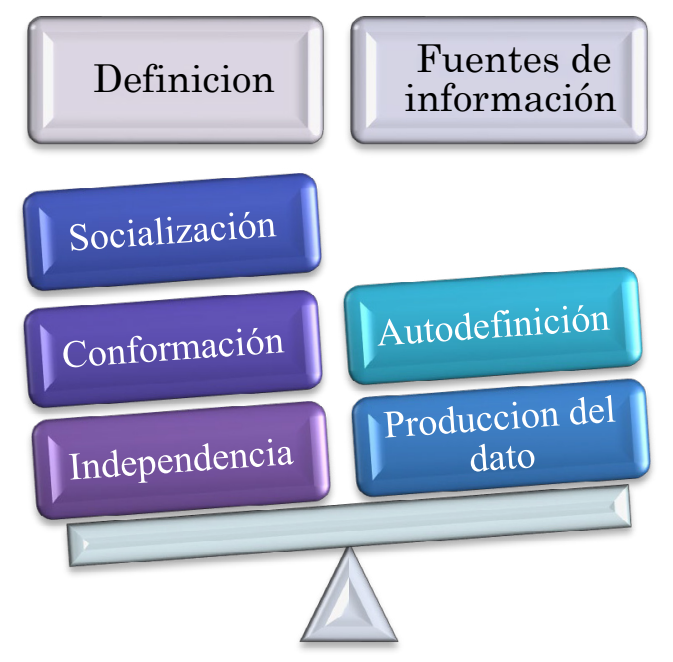

Figura 3. Problemas con respecto al tema de los observatorios.

Fuente: Elaboración propia a partir de Robles (2015).

A través de lo citado por Vessuri (2002, citado por De La Vega, 2007) los observatorios deben ser quienes tiene que abordar el tema de la información y su importancia con la ciencia, la tecnología y la innovación, es necesario vender la idea de la utilidad de los informes y como conseguir colaboración en los procesos de recolección de la información (p.550-551).

Ahora bien, los sistemas de información están entendidos según Peña (2006, citado por Duany, 2010) como "un conjunto de elementos interrelacionados con el propósito de prestar atención a las demandas de información de una organización, para elevar el nivel de conocimientos que permitan un mejor apoyo a la toma de decisiones y desarrollo de acciones" (p.1). Los sistemas de información son un elemento que hace parte importante de todo el proceso en el manejo de la información contable.

Las redes de conocimiento e información son generadoras de datos e información, si se logran mantener los flujos de estas por medio de las redes según Moreno y Del Pilar (2009), se estará cumpliendo con uno de los factores que permite la sostenibilidad en el tiempo de los procesos propios de un observatorio.

Con relación a la estructura en la organización del observatorio efectivamente cambia según el contexto donde se encuentre, deberán evolucionar de acuerdo a los cambios de su entorno, finalmente, los aprendizajes en las visitas efectuadas a los 
cuatro observatorios a nivel nacional, generan ideas valiosas de ilustración sobre detalles para el funcionamiento los cuales serán presentados en la última sección.

En palabras de Rodríguez (citado por Rivera-González y Rubiano-Aranzales, 2016), se menciona que es importante delimitar el espectro de observación, no es fácil determinar aproximadamente cuales son las principales líneas y tendencias contables de investigación a nivel nacional sobre las cuales se deba enfocar la observación, sin embargo con anterioridad a este artículo y en la primera parte del proyecto sobre tendencias en información contable en Colombia (Beltrán y González, 2017). Se generaron encuestas y revisión de revistas que dieron como resultado una propuesta en la identificación de 9 líneas de investigación como son: Auditoría, contabilidad ambiental y social, contabilidad de gestión, contabilidad financiera, contabilidad internacional, contabilidad pública, investigación y educación contable, teoría contable y tributación, más adelante se especifica cual es la temática escogida, el fin del observatorio seria tomar todas las temáticas, eso sería lo correcto, sin embargo no es tarea fácil el iniciar un proyecto de tal magnitud intentando desde el inicio organizar y cubrir todas las temáticas, es por esto que se propone iniciar tan solo con una o solo algunas de las anteriores líneas como foco de observación.

A continuación, se irán presentando cada una de las actividades que pueden conformar la estructura organizacional del observatorio contable, de igual manera una guía de ejemplo, de cómo podrían quedar cada una de ellas dentro de la estructura.

\section{Propuesta OBSERVATORIO DE INVESTIGACIÓN CONTABLE}

\section{Actividades generales propuestas}

El diseño estructural y de gestión de la propuesta debe tener en cuenta la teoría administrativa ya que de acuerdo a Reyes \& Ponce (2002) la organización es inherente en su mecánica a ayudar para estructurar y guiar en la generación de funciones, actividades y jerarquías, de esta manera también se revisa algo de lo presentado por Barrios (2009), quien estructura un esquema sencillo, el cual posee elementos representativos a tener en cuenta dentro de la estructura organizacional (Figura 4).

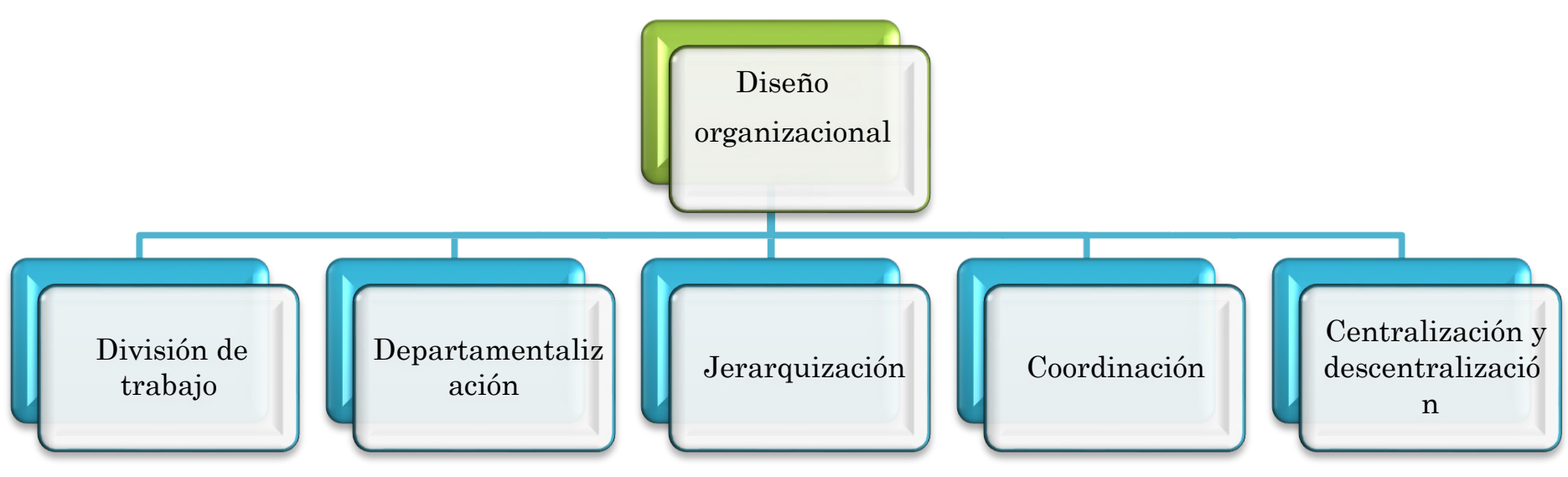

Figura 4. Elementos básicos de diseño organizacional. Fuente: Elaboración propia a partir de Barrios (2009). 
La distribución de labores y asignación acertada de las mismas se basa en el tipo de perfil que tendrán los integrantes, se generan áreas o departamentos con tareas propias, se ve necesaria también la existencia de un líder que coordine e impulse la consecución de los objetivos, de ellos depende integración de procesos y la sostenibilidad del proyecto.

Para Mintzberg (citado por Moreno y Del Pilar, 2009) los elementos que constituyen la estructura organización son parte del panorama de variables, se presta especial relevancia a la tecnología con la cual se va a trabajar, el entorno dentro de la empresa y la estrategia escogida para enfocar sus actividades, de tal forma son múltiples los aspectos internos como externos que se deben evaluar.

Es imperante como dentro de toda entidad el que exista un plan de indicadores operativos que midan las actividades así Francés (2006), aconseja que sean pocos y sencillos con el fin de conseguir su real seguimiento, pero que permitan medir el cumplimiento de cada una de ella, se proponen: a) Indicadores de Cantidad, b) de Calidad, c) Eficiencia o desempeño, sería importante consultar material sobre como reconocer los riesgos en los procesos, de este estudio seguramente se podrá plantear indicadores complementarios.

Según lo anterior se pueden agrupar las actividades y tareas en departamentos o secciones como parte del proceso administrativo, en el cual se proponen cuatro funciones básicas o divisiones dentro de la organización como son; desarrollo del talento humano, marketing, producción y operaciones, contabilidad y finanzas para poder tener control y tomar decisiones al respecto de los asuntos financieros, como resultado de la información que se recopile (Bernal, 2014; De la Torre, 2018). Esto no significa la imposibilidad en crear los departamentos, secciones o funciones acorde con las necesidades de la organización; ahora bien, se propone el siguiente diagrama por departamentos de acuerdo a las funciones o tareas agrupadas de forma similar, que se van a desarrollar dando origen a cada departamento así (Figura 5):

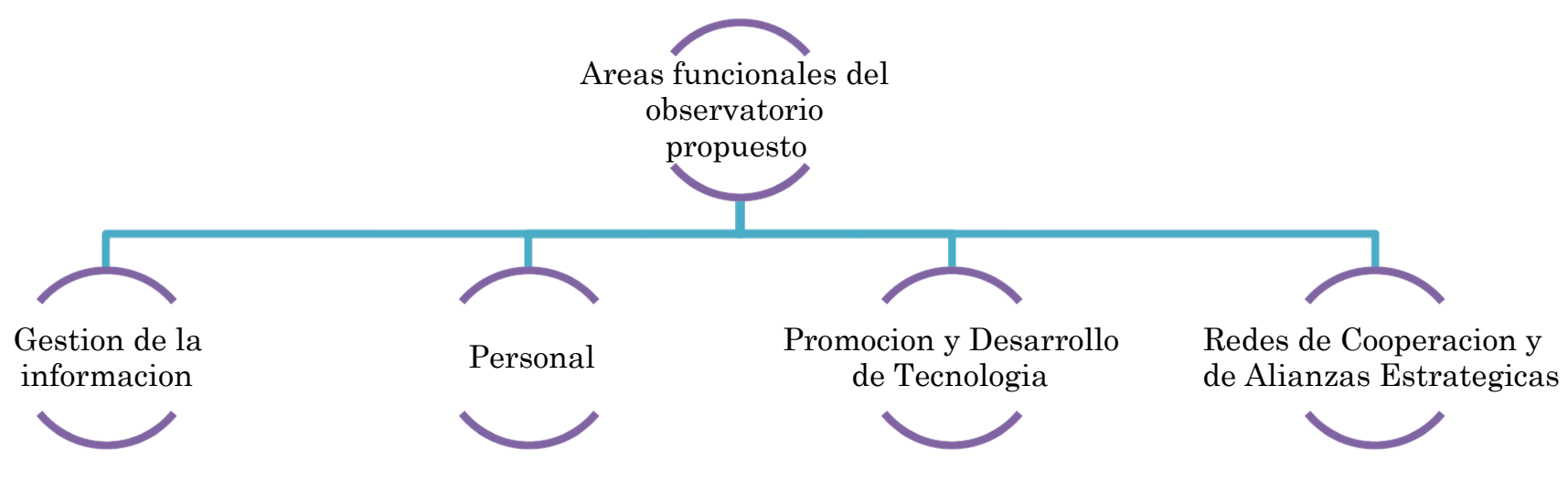

Figura 5. Estructura Inicial por Áreas Base del Observatorio. Fuente: Elaboración propia a partir de las entrevistas en observatorios nacionales.

Un área funcional es el conjunto de procesos, actividades y responsabilidades realizadas en un departamento o área de la organización (Münch, 2014). Por otra parte, para Franklin (2014), la Orientación a funciones, procesos o su combinación 
valora las condiciones que más favorecen el funcionamiento de la organización en lo relativo a la estructura, delegación de facultades, distribución de las unidades administrativas, formas de coordinarse y de generar bienes y servicio, la idea es poder precisar la jerarquía de la información y traducirla en procesos estratégicos (procesos); en cuanto a la interrelación de función/proceso, tratar de obtener el mayor provecho posible de la estructura funcional y de la gestión de la información, materia prima y energía de los procesos.

\section{Gestión de la información}

La información se conforma en el principal insumo generador de los documentos que son producto del Observatorio y también insumo clave para los investigadores a nivel nacional, estos deben ser generados con características tales como; útiles, agiles, breves, importantes y que sugieran alguna acción a seguir. "Una institución de información es una organización del conocimiento" que en este caso no es para su propio beneficio, porque esta es una entidad sin ánimo de lucro, pero si para el beneficio de la profesión y de la sociedad en general (Aja, 2002). La conexión de la información con todas las partes de la estructura tanto interna como externamente es una gran responsabilidad en su administración puesto que la información es la que permite guiar los procesos.

Algunas actividades dentro de la gestión de la información:

- Recepción y verificación de la información recibida de las redes.

- Generación de cronograma para recibo y producción de información.

- Fechas y reuniones semestrales de los integrantes del observatorio.

- Análisis y estructuración de las bases de datos.

\section{a. Investigación:}

Sucederá como algo inherente al hecho de manejar y profundizar en temas contables que dan cuenta de las tendencias en investigación contable en Colombia, se tratara de revisar y observar la realidad de lo que está pasando con la investigación en la temática seleccionada, esto es investigar (Zubiri, 2005), sea cualquiera el tema escogido en el área contable, se espera que los datos a analizar sean tanto cuantitativos como cualitativos por tanto la metodología será mixta, para llegar hasta el hecho de observar de la forma más objetiva posible y así informar el estado de la línea, en la propuesta aquí presentada se toma como ejemplo la línea de contabilidad Pública.

En este proceso de investigación se presentan algunas actividades a cumplir, como:

- Revisión de fondo de la información recibida de acuerdo a lo solicitado y acordado con los socios o redes de cooperación.

- Filtrar y escoger la información más relevante relacionada con nuestro tema observado. 
- Elaboración de estadísticas y generación de informes.

- Almacenamiento y archivo histórico de los informes.

\section{b. Productos del Observatorio:}

Los informes deben ser precisos, claros y confiables, al respecto (Cohen y Lares, 2004). Explica que la información debe poseer unas características claves como son: tiempo, contenido y forma, acorde a ello se espera centrarnos en el tema y de la mejor forma estructurar diferentes tipos de informes, entre ellos se proponen las siguientes orientaciones:

- Estudio por regiones

- Estudio de sectores económicos particulares

- Estudio de Actividades especificas

- Estudio temático

- Diagnósticos

Los anteriores puntos enfocados a el área y temática que haya sido seleccionada. Se puede generar actualización por medio de boletines informativos, las cuales deben ser periódicas en un lapso intermedio entre la presentación de los informes semestrales o anuales, se dejan estos informes en periodos que puedan suplir noticias importantes que tengan que ser presentadas prontamente. Por otra parte también se debe tener en cuenta según un estudio realizado por Tarazona y Martínez (2014) algunos de los principales aspectos para generar el éxito de una publicación, así mejorando el impacto y la visibilidad en varios campos, estos varían entre la pertinencia en la puntualidad de la publicación, el contenido del documento y su traducción a otros idiomas, esta información apoyaría la formación de redes, permitiendo establecer un diálogo permanente de intereses relacionados que se estén llevando a cabo en otras naciones. Es posible que los administradores del observatorio decidan o no el generar artículos de investigación, pero esto dependerá de las actividades que se definan.

\section{Estructura funcional, personal}

Toda organización independientemente de la naturaleza que sea, necesita del talento humano (recurso humano) para cumplir con sus objetivos. Para Chiavenato (2017) la administración incluye la coordinación de recursos humanos y materiales para lograr las metas. En esta concepción se configuran cuatro elementos básicos: 1) Alcance de los objetivos, 2) Por medio de personas, 3) Mediante técnicas y procesos, y 4) En una organización. Básicamente, la tarea de la administración es integrar y coordinar recursos organizacionales muchas veces cooperativos, otras veces en conflicto, como personas, materiales, dinero, tiempo, espacio, etc. 
Para poder desarrollar las actividades del observatorio contable, igualmente se propone la existencia del siguiente personal quien se hará cargo de los procesos, un ingeniero de sistemas que se encargara de la página web de su manejo y alistamiento de datos para su estudio, este podría ser contratado de medio tiempo o tiempo completo, también un Coordinador o Director del observatorio, el cual deberá ser preferiblemente de la disciplina contable y con un perfil de maestría o doctorado, el comité de investigadores y analistas de la información estaría conformado como mínimo por dos profesores los que estarían a cargo de elaboración de informes y manejo de información en el comité de análisis, se sugiere que estos dos sean de dedicación y tiempo completo, adjuntos a ellos se pueden invitar estudiantes que a modo de práctica o pasantía presten ayuda y se formen como futuros gestores del observatorio.

De la Fuente (1994) hace un estudio cuidadoso que relaciona estas tres variables, "contexto-estructura-eficiencia", cada organización es particular y por lo tanto su estructura también se debe adaptar de igual forma a sus objetivos a su entorno, con el fin de lograr lo esperado con buenos resultados, además no se debe dejar de lado otros insumos importantes como: equipos de cómputo, software y espacios de trabajo, otros gastos de funcionamiento como la papelería, impresoras etc., son elementos básicos pero igualmente importantes para que el observatorio pueda cumplir bien su función, se debe valorar y hacer una inversión que lleve la teoría administrativa ya citada, de los libros de administración a la práctica, sin dejar de perder de vista el objetivo principal o la misión, se reitera que este tipo de proyectos no se pueden medir por su rentabilidad, puesto que son con un fin social, académico y sin ánimo de lucro.

\section{La promoción y desarrollo tecnológico}

El impacto de la información se relaciona con su visibilidad y en el poder ser compartida con los grupos de interés (Rivera y Rubiano, 2016), el observatorio de hecho está obligado a poseer una página web, actualizada, con una buena publicidad, que sea de fácil acceso y manejo por parte de los usuarios, un ingeniero de sistemas se encargara de mantenerla actualizada y con excelente presentación, esta es la ventana del observatorio, el desarrollo tecnológico debe ser utilizado al máximo impactando por medio de su labor a través de los informes, la tecnología aplicada se utilizará para la revisión, ordenamiento y análisis de datos, la colaboración y difusión de la red de información creada en torno al tema contable se fortalecerá con las relaciones internas como externas generando impacto en nuestra área temática (Muñoz y Calvo, 1992) se puede no solo hablar de desarrollo tecnológico en la implementación del proyecto, sino también de innovación ya que es un proyecto inexistente en el país.

\section{Redes de Cooperación y de Alianzas Estratégicas}

Sin redes o acuerdos de colaboración para el suministro de información contable sobre el área de interés, es imposible mantener en funcionamiento el observatorio, pensar en cuales deben ser los socios de interés es uno de los principales problemas 
que ha surgido, debido a esto, se ha llegado a pensar que no necesariamente deben ser las universidades quienes sean las socias del proyecto y ni siquiera las precursoras en montar este observatorio, bien podría ser fundado por una universidad pero se sugiere que su socio de cooperación sea una entidad del estado, por ejemplo conseguir alianzas con el Ministerio de Educación, o con Colciencias, este último se puede pensar como uno de los más acertados, puesto que manejan los cvlac y los gruplac en todo el país y es allí donde encontramos las tendencias relacionadas con las líneas de investigación, investigadores, grupos, etc.

También se podría generar alguna relación con bases de publicaciones científicas periódicas como: Scopus, Procuest, o web of science que serían una buena e importante fuente de información en todo el territorio nacional, si se lograran estas alianzas se posibilitaría el continuo mantenimiento del flujo de entrada de información al sistema, por otro lado se sugiere que los acuerdos entre las partes deben ser a largo plazo.

En la red de cooperación cada uno de sus participantes debe tener claro cuáles serán sus obligaciones y alcances, cada participante debe entender que existen unos límites y que al final cada una de ellos se puede ver afectado positiva o negativamente por la otra parte (Fernández y Peña, 1999). El director del observatorio será el encargado de mantener la comunicación y unas relaciónese activas con los socios del proyecto.

\section{Compilatorio de la Propuesta de Estructura del Observatorio Contable}

En la siguiente Figura 6 se presenta el resumen completo de las áreas propuestas, se encuentra lo relacionado con principales las actividades y el personal, es de resaltar que esta es una propuesta flexible para quien este interesado y podrá ser complementada y/o ajustada en su desarrollo.

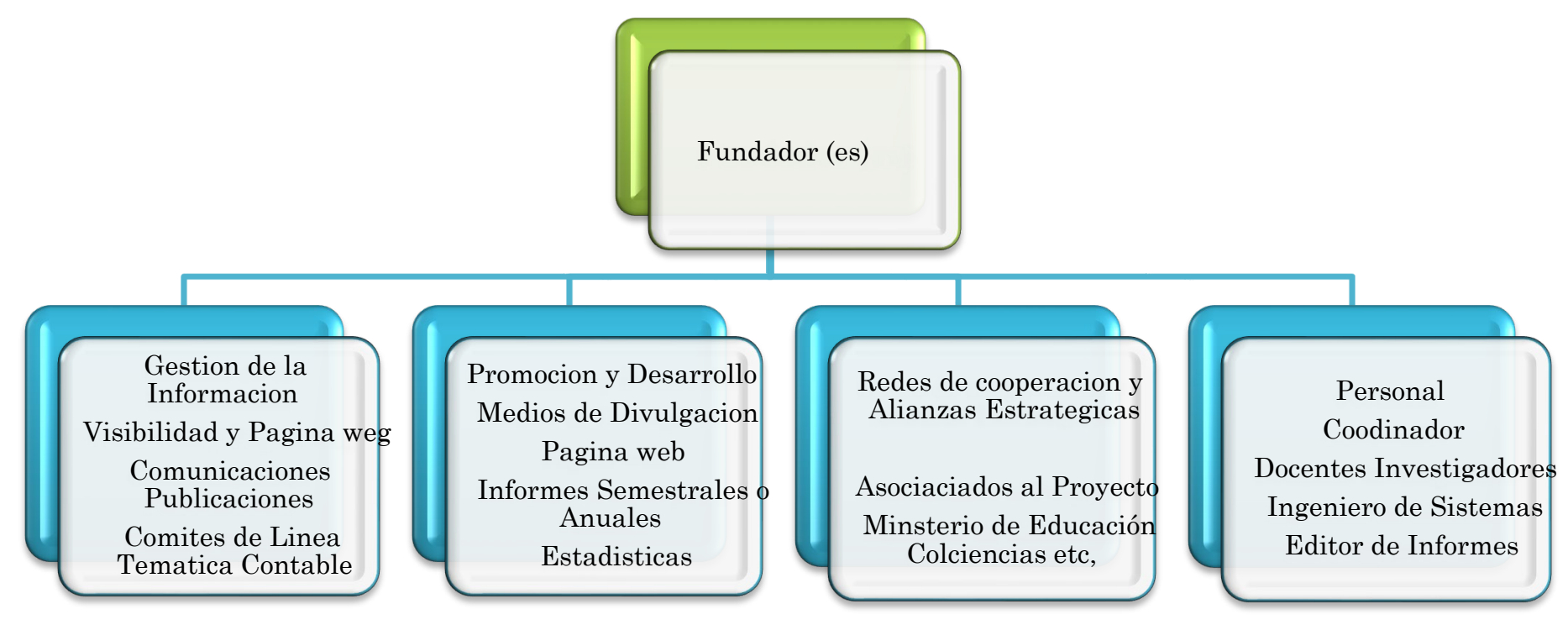

Figura 6. Compilatorio de la Propuesta de Estructura del Observatorio Contable. Fuente: Elaboración propia a partir de la investigación. 


\section{SUGERENCIAS Y CONSIDERACIONES}

Cómo se comentó anteriormente esta es una propuesta enfocada hacia la parte estructural, queda pendiente la propuesta de fortalecimiento para la parte estratégica que será desarrollada en otro artículo, teniendo en cuenta que los mínimos aquí contenidos deben ser tenidos en cuenta cuando se ponga en funcionamiento el observatorio, sin las redes de información y la alianza inicial, no tiene sentido el proyecto y no existirá el insumo para el funcionamiento y la producción o emisión de informes que dan sentido al servicio o fin último del proyecto, la especificación y claridad en la definición como observatorio guiara indefinidamente las funciones y fines para los que fue creado, el apoyo y credibilidad a las funciones del personal que lo constituye, es un impulso que debe ser entregado en todo momento.

Se debe tener en cuenta que, si se conoce la información completa y clara sobre las líneas de investigación contable, se podría promover el crecimiento de nuestra profesión, la evolución y conocimiento se basaría en nuevos desarrollos.

\section{Conclusiones}

Este proyecto puede ser iniciativa de cualquier organización interesada ya sean: universidades, empresas, grupos de investigación, etc. del ámbito particular o público, no necesariamente deberá ser aplicado por parte de la académica, es decir en las universidades, pero si puede ser una de las más opcionada y si logra consolidarse en una entidad de este tipo, la sugerencia es que se debe poseer un alto nivel de autonomía en sus decisiones.

El sector académico o científica, o cualquier tipo de interesado debe reconocer la importancia de una estructura más formal que posea cada una de las áreas necesarias para su buen funcionamiento, la publicidad y visibilidad en una página web es vital para su reconocimiento lo cual permitirá poder compartir la información sobre el área focalizada.

El aporte de la información en el área escogida será un aporte nuevo para los investigadores a nivel nacional, seguramente se podrá ir fortaleciendo con el fin de ir cubriendo otras áreas contables de interés que puedan ir solicitando información a nivel nacional.

Como a nivel nacional y contable no existen en este momento proyectos de este tipo, todo lo que se presente en el montaje y puesta en marcha será aprendizaje, lo cual seguramente será aporte y experiencia a la disciplina contable y para otras ciencias como la administrativa o económica.

Definitivamente al ser una entidad sin ánimo de lucro genera una resistencia para que algún interesado emprenda un proyecto de esta magnitud, el conocimiento generado por la gestión en información será el aporte a la contaduría y a la ciencia e investigación contable a nivel nacional, el aporte no solo a la academia sino también a la economía, a los sectores público y real, será un impacto real a futuro siendo un producto colateral de la investigación en las áreas sobre las cuales se genere información, un efecto en cadena producto de las investigaciones relacionadas con la línea contable escogida. 
Los tiempos deben quedar claramente estipulados en los cronogramas de trabajo, al iniciar los procesos se asignarán detalladamente las actividades y responsabilidades reconociendo claro está, que es un proceso más académico que administrativo.

La productividad en artículos, informes, noticias debe ser de excelente calidad con los parámetros que se deben tener en cuenta según las indicaciones sugeridas, este puede ser un punto de quiebre que genere una buena o mala imagen en la sociedad del conocimiento, por lo tanto, los primeros reportes son clave y deberán medir el resultado y la aceptación por parte de los stakeholders tanto internos como externos de todos los sectores.

Existe la posibilidad que los contadores públicos logremos integrarnos alrededor de una información focalizada, serán el medidor de los resultados, generando nuevas propuestas en torno al conocimiento activo de nuestra disciplina y en particular al tema de interés.

\section{Reconocimientos y AgRadecimientos}

Artículo producto de investigación científica y tecnológica del programa de Contaduría Pública de la Facultad de Estudios a Distancia, apoyado por la Universidad Militar Nueva Granada, vigencia 2019.

\section{REFERENCIAS}

Acurero, M., Ziritt, G. Y. y Chumaceiro, A. (2017). Tecnologías de información y comunicación: una visión desde la universidad y la integración laboral en América Latina y el Caribe. En, R. Prieto \& J. García (Comp). Gerencia Empresarial. Una perspectiva integral desde lo público y privado (pp. 167-193). Barranquilla: Ediciones Universidad Simón Bolívar. Disponible en http://bonga.unisimon.edu. co/bitstream/handle/20.500.12442/3219/GerenciaEmpresarial.pdf?sequence=1 \&isAllowed=y\#page $=168$

Aja, L. (2002). Gestión de información, gestión del conocimiento y gestión de la calidad en las organizaciones. Acimed, 10(5), 7-8. Disponible en http://biblioteca. udgvirtual.udg.mx/jspui/handle/123456789/893

Barrios, D. (2009). Diseño organizacional bajo un enfoque sistémico para unidades empresariales agroindustriales. [Tesis magistral]. Universidad Nacional de Colombia. Medellín, Colombia. Disponible en http://bdigital.unal.edu.co/1901/

Bejarano, H. D., González, L. y Matallana, A. (2017). Componentes y variables de los observatorios contables existentes a nivel superior en Colombia. Aglala. 8(1), 84-116. Disponible en http://revistas.curnvirtual.edu.co/index.php/aglala/article/view/1027

Beltrán, J. P. y González, L. A. (2017). Tendencias de Investigación Contable en Colombia: Teoría o Práctica, Una Mirada desde las Revistas Contables Colombianas 2011 a 2015. Face, 17(2), 68-80. Disponible en http://revistas.unipamplona. edu.co/ojs_viceinves/index.php/FACE/article/view/2228

Bernal, C.A. (2014). Introducción a la administración de las organizaciones. Nueva Jearsey: Pearson. 
Bianco, C., Lugones, G., Peirano, F. y Salazar, M. (2016). Indicadores de la Sociedad del Conocimiento: aspectos conceptuales y metodológicos. Bogotá,D.C.: Colciencias. Recuperado de http://hdl.handle.net/11146/158

Carrera, N. P. y López, N. S. (2013). Observatorio de turismo. Un espacio de análisis y Comunicación como contribución para el desarrollo del Turismo en el Ecuador. Gestión Turística, (19), 9-24. https://doi.org/10.4206/gest.tur.2013.n19-02

Chiavenato, I. (2017). Administración de recursos humanos. Bogotá, D.C.: Mc-Graw Hill.

Chiavenato, I. (2017). Comportamiento organizacional. Santa Fe de Bogotá: McGraw Hill. Recuperado de http://ezproxy.umng.edu.co:2048/login?url=http:// www.ebooks7-24.com/?il=5205

Cohen, K. D. y Lares, E. (2004). Sistemas de Información para los negocios. México, D.F.: Mc-Graw Hill.

De la Fuente, J. M. (1994). Estructura organizativa y eficiencia empresarial: propuesta de un modelo contingente de análisis. Ekonomiaz: Revista vasca de economía, (30), 178-197. Disponible en https:/www.euskadi.eus/ekonomiaz/

De la Torre, M. (2018). Utilización del sector financiero para el lavado de dinero: perspectiva desde la legislación ecuatoriana. Juridicas CUC, 14(1), 145-166. http://dx.doi.org/10.17981/ juridcuc.14.1.2018.7

De la Vega, I. (2007). Tipología de Observatorios de Ciencia y Tecnología. Los casos de América Latina y Europa. Revista española de documentación científica, 30(4), 545-552. Recuperado de http://redc.revistas.csic.es/index.php/\%20redc/ article/viewFile/404/416

Donati, P. (1997). El desarrollo de las organizaciones del Tercer Sector en el proceso de modernización y más allá, Reis, (79), 113-141. Recuperado de http://reis.cis. es/REIS/PDF/REIS_079_06.pdf

Duany, A. (2010). Sistemas de Información. [Online]. Disponible en https://www. econlink.com.ar/sistemas-informacion/definicion

Fernández, J. C. y Peña, N. (1999). Las redes de cooperación empresarial: ¿Una organización para el próximo milenio? Dirección y Organización, (21), 12-19. Disponible en https://www.revistadyo.es/DyO/index.php/dyo/article/view/289/289

Francés, A. (2006). Estrategia y planes para la empresa: con el cuadro de mando integral. México, D.F.: Pearson Educación.

Franklin, E. B. (2014). Organización de empresas. México, D.F.: Mc-Graw Hill.

González, F. M. (2007). Introducción a los Sistemas de Información: Fundamentos. [Online]. Disponible en http://metabase.uaem.mx//handle/123456789/2471

Hernández, J., Melendez, Y., Chumaceiro, A. y Aguilar, A. (2017). Calidad y cultura educativa. Tendencias de la inclusión en el siglo XXI. En, J. Hernández, J. Barboza e I. Muñoz (Comp.). Calidad de Vida, Inclusión Social y Bienestar Humano. (pp. 6-22). Cabimas: UNERMB. https://doi.org/10.21892/9789804270215

Hernández, M. y Buitrago, R. (2017). Rol del sinésteta organizacional en el manejo de la comunicación asertiva. Económicas CUC, 38(2), 61-76. https://doi. org/10.17981/econcuc.38.2.2017.05

Machado, M. A. y Zapata, M. Á. (2016). Modelación e información contable para entornos Competitivos. Contaduría Universidad de Antioquia, (36), 149-168. Disponible en https://revistas.udea.edu.co/index.php/cont/article/view/25564 
Méndez, J. A., Jiménez, M. G. y Cervantes, M. L. (2016). Retos y desafíos de las redes de investigación. REICE. Revista Iberoamericana sobre Calidad, Eficacia y Cambio en Educación, 10(3), 178-182. Disponible en https://revistas.uam.es/ reice/article/view/3051

Moreno, C. F. y Del Pilar, L. A. (2009). La estructura organizacional y el diseño organizacional, una revisión bibliográfica. Gestión \& Sociedad, 2(1), 97-108. Disponible en http://bit.ly/2hBm3yh

Moyares, Y. M. e Infante, M. B. (2016). Caracterización de los observatorios como plataformas para la gestión de la vigilancia tecnológica en el sector de la Educación Superior. Enlace, 13(1), 11-27. Disponible en https://produccioncientificaluz.org/index.php/enlace/article/view/21101/20953

Müller, R. (2010). Manual para la presentación de proyectos. Lima: FondoEmpleo. Recuperado de http://fondoempleo.com.pe/Dir_Sistemas_FE/OLD/11_C/B/Man11Conc_L4.pdf

Münch, L. (2014). Fundamentos de gestión empresarial: La clave de la competitividad. Administración. México, D.F.: Trillas.

Muñoz, M. P. y Calvo, L. (1992). El desarrollo tecnológico: un reto para la contabilidad. Revista Española de Financiación y Contabilidad, (71), 329-346. Recuperado de https://www.macam.es/leandro/articulos/EL\%20DESARROLLO\%20 TECNOLOGICO.pdf

Observatorio Colombiano de Educación Contable. (2012). La problemática de la educación contable en Colombia: las perspectivas para la construcción del Observatorio Colombiano de Educación Contable. Activos, 10(19), 91-109. https://doi. org/10.15332/s0124-5805.2012.0019.04

OCDE. BID. (2016), Impulsando la productividad y el crecimiento inclusivo en Latinoamerica, Santiago de Chile: OCDE \& BID.

Ortegón, E., Pacheco, J. F. y Prieto, A. (2005). Metodología del marco lógico para la planificación, el seguimiento y la evaluación de proyectos y programas. New York: United Nations Publications.

Paredes-Chacín, J. (2017). Planificación financiera ante la perspectiva organizacional en empresas cementeras del estado Zulia-Venezuela. Económicas CUC, 38(1), 105-132. https://doi.org/10.17981/econcuc.38.1.05

Paz, A., Harris, J. y Franco, F. (2016). Responsabilidad social gestión compartida con el emprendedor social en empresas mixtas del sector petrolero. Económicas CUC, 37(2), 47-68. https://doi.org/10.17981/econcuc.37.2.2016.03

Pinzón, J. E. (2012). Sobre una conversación incidental el Observatorio Colombiano de Educación Contable, OCEC. Cuadernos de Contabilidad, 13(32), 243249. Disponible en https://revistas.javeriana.edu.co/index.php/cuacont/article/ view/3899

Ravina-Ripoll, R., Ahumada-Tello, E. y Gálvez-Albarracín, E. J. (2019). La felicidad como predictor del rendimiento académico de los estudiantes universitarios. un análisis comparativo entre México y España. Cauriensia. Revista Anual De Ciencias Eclesiásticas, 14, 407-426. Disponible en https://www.cauriensia.es/ index.php/cauriensia/article/view/EM10 
República de Colombia. Presidencia de la República. (2012). Bases para la construcción de observatorios territoriales de juventud, Programa presidencial para el sistema nacional de juventud (Colombia Joven). Bogotá, D.C.: Presidencia de la República. Recuperado de https://dapre.presidencia.gov.co/AtencionCiudadana/ DocumentosCiudadania/bases-construccion-observatorios-territoriales-juventud.pdf

República de Colombia. Presidencia de la República. (2006). Guía para la conformación de Observatorios de DD. y HH. Bogotá, D.C.: Impresol. Recuperado de http://2014.derechoshumanos.gov.co/Observatorio/Publicaciones/Documents/2010/manualguia.pdf

Reyes, A. y Ponce, A. R. (2002). Administración de empresas: Teoría y Práctica / Business Administration. México, DF: Limusa.

Rivera-González, M. Á. y Rubiano-Aranzales, E. (2016). El observatorio, una herramienta para el sector social, cooperativo y solidario en la región Tolima. Cooperativismo \& Desarrollo, 24(109), 119-133. https://doi.org/10.16925/co.v24i109.1510

Robles, R. S. (2015). ¿Qué es un Observatorio Ciudadano? Vinculos. Sociología, análisis y opinión, (4), 189-212. Disponible en http://www.vinculossociologiaanalisisyopinion.cucsh.udg.mx/index.php/VSAO/article/view/4186

Soler, J. y Catalá, C. (noviembre, 2007). Observatorio SIC: análisis de una iniciativa de comunicación basada en el expertise del servicio de información y conocimiento de ESADE, 2007. Paper presentado en la conferencia, IX Jornadas de Gestión de la Información, ESADE, Madrid. Recuperado de http://eprints.rclis. org/10843/

Tarazona, A. H. y Martínez, J. P. (2014). Consecuencias de la importancia de modelos de indexación para medir la producción académica nacional. Revista de Educación y Desarrollo Social, 8(1), 70-83. Disponible en https://revistas.unimilitar. edu.co/index.php/reds/article/view/588

Torres, A. R. y Martínez, J. C. (2014). Análisis y propuesta de implementación de un observatorio TIC para un conjunto de mipymes de la localidad de Usaquén (Bogotá) en la Universidad de San Buenaventura. Ingenium Revista de la Facultad de Ingeniería, 15(29), 124-147. https://doi.org/10.21500/01247492.1349

Vallejo, A. Echavarría, M. A. y Uribe, M. A. (2009). Observatorios y redes de cooperación internacional. Negocios Internacionales, 2(1), 52-66. Recuperado de http://publicaciones.eafit.edu.co/index.php/rni/article/view/306

Zubiri, X. (2005). ¿Qué es investigar? The Xavier Zubiri Review, 7, 5-7. Recuperado de http://www.zubiri.org/general/xzreview/2005/pdf/xzr_2005.pdf

\section{BIODATA}

Luis Alfonso González Feliciano es Contador Público. Magister en Educación. Especializado en Docencia Universitaria y Control Interno. Docente tiempo completo e integrante del grupo de investigación INCONDIS del programa de Contaduría Pública de la Facultad de Estudios a Distancia de la Universidad Militar Nueva Granada (Colombia). https://orcid.org/0000-0003-0470-0752 